\title{
VOLATILE CONSTITUENTS OF THE AERIAL PARTS OF TERATAENIUM LASIOPENTALUM (BOISS.) MANDEN., STEMS AND LEAVES OF DOREMA AMMONIACUM D.DON. AND LEAVES, FRUITS AND STEMS OF LEUTEA PETIOLARE (DC.) M.PIMEN FROM IRAN
}

\author{
SHIVA MASOUDI ${ }^{1, *}$, SAHAR KAKAVAND ${ }^{l}$ \\ ${ }^{1}$ Department of Chemistry, Faculty of Science, Islamic Azad University, Central Tehran Branch, P. O. Box 13185-768, Tehran, Iran
}

\begin{abstract}
The Composition of the essential oils from three Umbelliferae species of Iran, Tetrataenium lasiopetalum (Boiss.) Mandem ., Dorema ammoniacum D.Don., which is endemic to Iran, and Leutea petiolare (DC.) M.Pimen. obtained by hydrodistillation were analyzed by GC and GC/MS. Germacrene D (17.72\%) and (E,Z) farnesol (12.58\%) were the main components in the oil of aerial parts of T. lasiopetalum. The major compound in the stem oil of D. ammoniacum was $\alpha$-muurolol $(13.68 \%)$, whereas the leaf oil contained (E)- $\beta$-ocimene $(30.94 \%), \gamma$-terpinene $(11.09 \%)$, p-cymene $(10.03 \%)$ and $(\mathrm{Z})$ - $\beta$-ocimene $(7.11 \%)$. Terpinolene $(13.93 \%$, $8.28 \%$ and $10.27 \%)$, (E)- $\beta$-ocimene $(10.24 \%, 24.63 \%$ and $10.46 \%)$ and endo-fenchyl acetate $(15.1 \%, 7.42 \%$ and $7.57 \%)$ were the main constituents in the leaf, fruit and stem oils of $\mathrm{L}$. petiolare respectively.
\end{abstract}

Keywords: Tetrataenium lasiopetalum, Dorema ammoniacum, Leutea petiolare, Umbelliferae, Essential oil

\section{INTRODUCTION}

The genus Tetrataenium is represented in Iran by two species. T. lasiopetalum (Boiss.) Manden. and T. nephrophyllum (Leutea) Manden., ${ }^{1,2}$ No studies on the chemical composition or oils of Tetrataenium species have previously been reported.

The genus Dorema is represented in the flora of Iran by six species, among which two are endemic: D. aucheri Boiss. and D. ammoniacum D. Don. ${ }^{1,2}$ D. ammoniacum is a perennial plant growing up to $2.5 \mathrm{~m}$ in height in arid and semi-arid regions of central Iran, such as Yazd, Isfahan and Semnan provinces. ${ }^{1}$

This species excudes a medicinal gum resin, commonly known as "Ushaq" or "Vasha" in Iranian traditional medicine and also as "Persian ammoniacum" in Greek and Latin medicinal literature.-5 In Iranian folk medicine, this gum resin has been considered useful in treatment of spastic pains, gastric disorders, intestinal parasitic infections and skin inflammations and as analgestic, stimulant, expectorant and laxative..$^{3,6-8}$ It has also been traditionally used in Western and Indian medicines as antispasmodic, expectorant, diaphoretic and emmeragogue and also for treatment of catarrh, asthma, chronic bronchitis and persistent coughing. ${ }^{9}{ }^{10} \mathrm{D}$. ammoniacum gum resin has been reported to exhibit some bioactivities including antibacterial, antifungal and acetylcholinesterase inhibitory effects. ${ }^{11-13}$.

Free salicylic acid, ammoresinol, ashamirone and some sesquiterpene chroman diones have also been isolated and identified from Persian ammoniacum from Iran. ${ }^{12-14}$ Presence of phenolic compounds such as sesquiterpene coumarins, phenols, flavonoids and phloroacetophenone glycosides have been reported from the other Dorema species. ${ }^{15-18}$.

There are also some reports about the essential oils obtained from D. ammoniacum from Iran. ${ }^{19-23}$.

The major constituents of the fruits oil of D. ammoniacum, collected from Semnan road toward Firooskuh, were (Z)-ocimenone (22.3\%) and (E)ocimenone (18.1\%). The results of the antimicrobial assay of the oil indicated that the oil exhibited moderate to high antimicrobial activity, especially against Bacillus subtillis and Staphylo coccusepidermidis. ${ }^{19-20 .}$

The oil from the leaves of the plant contained $\alpha$-gurjunene $(49.5 \%)$ and $\beta$-gurjunene $(19.0 \%)$ as the major components. ${ }^{21}$

The essential oils obtained by hydrodistillation of the flowers, stem and roots of D. ammoniacum collected from Hezar mountain in Rayen area, Kerman Province, has been reported. The major components in the flower oils were $\delta$-cadinene $(11.58 \%)$ and $\alpha$-himachalene $(7.71 \%)$. The stem oils contained $\delta$-cadinene $(16.24 \%)$, liguloxide $(8.69 \%)$ and $\delta$-amorphene $(8.43 \%)$ as the major components, while the root oil had 3-n-butyl phthalide $(62.49 \%)$, benzyl butanoate $(6.57 \%)$ and liguloxide $(5.15 \%)$ as the major components. The oils of flower, stem and root were richer in sesquiterpenes than monoterpenes..$^{22}$ Recently antioxidant and antibacterial activities of the essential oils and extracts from the aerial parts and roots of D. ammoniacum, collected from Kashan region have been reported. $\beta$-Himachalene $(9.3 \%)$ and $\beta$-chamigrene $(8.7 \%)$ were the main constituents in the aerial parts of the plant whereas $\beta$-bisabolene $(15.1 \%)$ and hexadecanal $(13.2 \%)$ were dominated in the roots oil. Ethyl acetate extract of the roots showed the highest antioxidant activity in both DPPH and FRAP assays. In antibacterial assay, the ethyl acetate and chloroform extracts of the roots exhibited strong antibacterial activity against Bacillus subtilis, Pseudomonos aeruginosa and Staphylococcus aureus. Essential oils were also found to be active against Shigella dysenteriae. ${ }^{23}$ According to M. Pimen point of view the Leutea genus is represented in Iran by five species: L. cupularis (Boiss.) M. Pimen., L. gracillima M. Piman., L. nematoloba (Rech. F.)., L. petiolare (DC.) M. Pimen and L. polycias (Boiss.) M. Pimen. ${ }^{1 .}$

In this work, we report the analysis of the essential oils of the aerial parts of Tetrataenium lasiopetalum, stems and leaves of Dorema ammoniacum and leaves, fruits and stems of Leutea petiolare, all found growing wild in Iran.

\section{EXPERIMENTAL}

Plant material: The aerial parts of Tetrataenium lasiopetalum was collected from Kellar mountain, Province of Chaharmahal-Bakhtiyari, west of Iran, in July 2013. The stems and leaves of Dorema ammoniacum, which is endemic to Iran, and the leaves, fruits and stems of Leutea petiolare both were collected from Shahroud, Province of Semnan in July 2013. Voucher specimens have been deposited at the Herbarium of the Research Institute of Forests and Rangelands (TARI), Tehran, Iran. Isolation of the essential oils: Air-dried aerial parts of Tetrataenium lasiopetalum $(120 \mathrm{~g})$, stems $(85 \mathrm{~g})$ and leaves $(70 \mathrm{~g})$ of Dorema ammoniacum and leaves $(70 \mathrm{~g})$, fruits $(60 \mathrm{~g})$ and stems $(90 \mathrm{~g})$ of Leutea petiolare were separately subjected to hydrodistillation using a Clevenger-type apparatus for $3 \mathrm{~h}$. After decanting and drying of the oils over anhydrous sodium sulfate, the corresponding yellowish coloured oils were recovered [in a yield of $0.2 \%, 0.3 \%, 0.7 \%, 0.4 \%, 0.2 \%$ and $0.1 \%(\mathrm{w} / \mathrm{w})]$, respectively. Gas chromatography : GC analysis was performed on Schimadzu 15 A gas chromatograph equipped with a split/splitless injector $\left(25^{\circ} \mathrm{C}\right)$ and a flame ionization detector $\left(250^{\circ} \mathrm{C}\right)$. Nitrogen was used as a carrier gas $(1 \mathrm{~mL} / \mathrm{min})$ and the capillary column used was DB-5 $(50 \mathrm{~m} \times 0.2 \mathrm{~mm}$, film thickness $0.32 \mu \mathrm{m})$. The column temperature was kept at $60{ }^{\circ} \mathrm{C}$ for $3 \mathrm{~min}$ and then heated to $220{ }^{\circ} \mathrm{C}$ with a $5{ }^{\circ} \mathrm{C} / \mathrm{min}$ rate and kept constant at $220{ }^{\circ} \mathrm{C}$ for 5 min. Relative percentage amounts were calculated from peak area using a Schimadzu C-R4A chromatopac without the use of correction factors. Gas chromatography-mass spectrometry : GC/MS analysis was performed using a Hewlett-Packard 5973 MSD detector with a HP-5 MS column (30 $\mathrm{m} \times 0.25 \mathrm{~mm}$, film thickness $0.25 \mu \mathrm{m})$. The column temperature was kept at $60{ }^{\circ} \mathrm{C}$ for $3 \mathrm{~min}$ and programmed to $220{ }^{\circ} \mathrm{C}$ at a rate of $5{ }^{\circ} \mathrm{C} / \mathrm{min}$ and kept constant at $220^{\circ} \mathrm{C}$ for $5 \mathrm{~min}$. The injector and GC/MS interphase were maintained at $270{ }^{\circ} \mathrm{C}$. The flow rate of helium, as carrier gas, was $1(\mathrm{~mL} /$ $\mathrm{min}$ ), with a split ratio of $1 / 50$. The ionization voltage was $70 \mathrm{eV}$. The ion source temperature was $250{ }^{\circ} \mathrm{C}$, and the transfer line temperature was $280{ }^{\circ} \mathrm{C}$. The mass range $(\mathrm{m} / \mathrm{z})$ was $45-465 \mathrm{amu}$ at a speed of $2.8 \mathrm{scan} / \mathrm{s}$. The retention indices for all the components were determined according to the Van Den Dool method, using n-alkanes as standards. The compounds were identified (RRI, DBS) by comparison with data reported in the literature and by comparison of their MS with either the Wiley library or with published MS. ${ }^{24,25}$ 


\section{RESULTS AND DISCUSSION}

The composition of the essential oils from aerial parts of Tetrataenium lasiopetalum, stems and leaves of Dorema ammoniacum and leaves, fruits and stems of Leutea petrolare are listed in Table 1,2 and 3, respectively, in which the percentage and relative retention indices of compounds are given.

As it is shown from Table 1, about $90.81 \%$ (48 components) of the oil of T. lasiopetalum were identified. The oil of the plant consists of five monoterpene hydrocarbons (5.79\%), eight oxygenated monoterpenes (6.75\%), fifteen sesquiterpene hydrocarbons $(43.21 \%)$, thirteen oxygenated sesquiterpenes $(22.34 \%)$ and seven non terpenoid compounds $(12.72 \%$.

Germacrene D (17.72\%) and (E,Z)-farnesol $(12.58 \%)$ were the major components in this oil. As can be seen from the above information in the aerial parts oil of T. lasiopetalum sesquiterpenes $(65.55 \%)$ predominated over monoterpenes $(12.54 \%)$ and other compounds $(12.72 \%)$.

As it is shown from the Table 2, sixty-four constituents $89.2 \%$ were identified in stem oil of D. ammoniacum: three hydrocarbon monoterpenes $(0.62 \%)$, five oxygenated monoterpenes $(6.93 \%)$, ten sesquiterpene hydrocarbons $(14.34 \%)$, thirteen oxygenated sesquiterpenes $(37.89 \%)$ and thirty-two non terpenoid compounds (29.42\%). $\alpha$-Muurolol (13.68\%) was the most abundant constituent followed by hexadecanoic acid $(6.81 \%)$ and $(\mathrm{E})$ nerolidol (5.09\%).

Twenty-five constituents $100 \%$ were identified in leaf oil: eight monoterpene hydrocarbons $(69.49 \%)$, five oxygenated monoterpenes $(10.45 \%)$, six sesquiterpene hydrocarbons $(10.26 \%)$, four oxygenated sesquiterpenes $(7.94 \%)$ and two non terpenoid compounds $(1.86 \%)$.

It was characterized by (E)- $\beta$-ocimene (30.94\%), $\gamma$-terpinene $(11.09 \%)$, p-cymene $(10.03 \%)$. Other notable constituents were $(\mathrm{Z})-\beta$-ocimene $(7.11 \%)$, terpinolene $(6.19 \%)$ and endo-fenchyl acetate $(5.25 \%)$.

The qualitative and quantitative variation between our results and previous reports for the concentration of the oils from stems and leaves of the plant may be attributed to the different environmental conditions. ${ }^{21-22}$

Previously we reported the main compounds among the 35 constituents characterizedin D. aucheri, representing $89.2 \%$ of the total components detected. ${ }^{26}$ Essential oil of D. glabrum roots have previously reported to contain $\delta$-cadinene (12.8\%), $\beta$-bisabolene (7.5\%) and $\alpha$-fenchyl acetate $(6.3 \%)$ as the main compounds. ${ }^{2}$

From Table 3, it is evident that forty-four components representing $91.05 \%$, fifty-three constituents representing $98.03 \%$ and thirty-six compounds representing $94.6 \%$ were identified in the oils of leaf, fruit and stem of Leutea petiolare respectively.

The main components in all oils were terpinolene $(13.93 \%, 8.28 \%$ and $10.27 \%)$, (E)- $\beta$-ocimene $(10.24 \%, 24.63 \%$ and $10.46 \%)$ and endo-fenchyl acetate $(15.1 \%, 7.42 \%$ and $7.57 \%)$, respectively. Other notable compounds were in leaf oil: limonene $(9.16 \%)$ and $(\mathrm{Z})-\beta$-ocimene $(7.79 \%)$, in fruit oil: p-cymene $(11.5 \%), \gamma$-terpinene $(9.72 \%)$ and $(\mathrm{Z})$ - $\beta$-ocimene $(8.32 \%)$ and in stem oil: p-cymene $(15.43 \%)$, hexadecanoic acid $(9.01 \%)$ and $\gamma$-terpinene $(5.27 \%)$.

As can be seen from the above information, the composition of the leaf, fruit and stem oils of L. petiolare were quite similar. All three oils were rich in regard to monoterpenes $(71.85 \%, 82.07 \%$ and $73.53 \%)$, respectively. The sesquiterpene fraction of the oils was relatively small, representing $(16.98 \%$, $14.51 \%$ and $7.96 \%$ ) of the total oils, respectively.

Previously we reported the essential oil from the aerial parts of $\mathrm{L}$. elbursensis Mozaffarian. Thirty-three compounds were identified in the oil of L. elbursensis, representing $98.0 \%$ of the total oil, with $\alpha$-pinene $(37.3 \%)$ and $\beta$-pinene $(36.1 \%)$ as the main constituents. The oil was rich in monoterpenes rather than sesquiterpenes. ${ }^{28}$

\section{CONCLUSIONS}

This paper presents the chemical composition of the essential oils of the aerial parts of Tetrataenium lasiopetalium, stems and leaves of Dorema ammoniacum and leaves, fruits and stems of Leutea petiolare. In the aerial parts oil of T.lasiopetalum sesquiterpenes predominated over monoterpenes. The composition of the stem oil of D. ammoniacum showed differentially to the leaf oil for the concentration of main compounds, also the stem volatile was rich in sesquiterpenes, while the leaf volatile was rich in monoterpenes. The leaf, fruit and stem oils of L. petiulare were rich in regard to monoterpenes.

\section{ACKNOWLEDGMENTS}

We are grateful to Dr. V. Mozaffarian (Research Institute of Forests and Rangelands, Tehran) for helpful assistance in collection plant material and for botanical identification.

Table 1: Chemical composition of essential oil from aerial parts of Tetrataenium lasiopetalum.

\begin{tabular}{|c|c|c|}
\hline Compound & $\mathrm{RI}^{\mathrm{a}}$ & T. lasiopetalum \\
\hline Hexanal & 800 & 0.15 \\
\hline 3-Nonene & 893 & 0.10 \\
\hline Nonane & 899 & 0.17 \\
\hline$\alpha$-Pinene & 939 & 1.14 \\
\hline Sabinene & 976 & 0.32 \\
\hline$\beta$-Pinene & 980 & 1.06 \\
\hline Car-3-ene & 1011 & 1.58 \\
\hline 1,8-Cineole & 1033 & 1.85 \\
\hline Terpinolene & 1088 & 1.69 \\
\hline iso-Pentyl isovalerate & 1103 & 8.53 \\
\hline Hexyl isobutyrate & 1150 & 1.73 \\
\hline Pinocarvone & 1162 & 0.18 \\
\hline Lavandulol & 1166 & 0.12 \\
\hline Terpin-4-ol & 1177 & 1.86 \\
\hline$\alpha$-Terpineol & 1189 & 0.48 \\
\hline Carvone & 1242 & 1.06 \\
\hline Lavandulyl acetate & 1289 & 1.06 \\
\hline Thymol & 1290 & 0.14 \\
\hline$\delta$-Elemene & 1339 & 0.18 \\
\hline Neryl acetone & 1365 & 0.24 \\
\hline$\alpha$-Copaene & 1376 & 0.16 \\
\hline$\beta$-Bourbonene & 1397 & 3.13 \\
\hline$-\alpha$ Gurjunene & 1409 & 2.17 \\
\hline$\beta$-Cedrene & 1415 & 4.08 \\
\hline$\beta$-Gurjunene & 1432 & 1.96 \\
\hline Aromadendrene & 1439 & 0.26 \\
\hline (E)- $\beta$-Farnesene & 1458 & 0.65 \\
\hline Germacrene D & 1480 & 17.72 \\
\hline Viridiflorene & 1493 & 3.59 \\
\hline$\alpha$-Muurolene & 1499 & 0.52 \\
\hline$(\mathrm{E}, \mathrm{E})-\alpha$-Farnesene & 1508 & 3.29 \\
\hline$\delta$-Cadinene & 1524 & 1.86 \\
\hline$\beta$-Sesquiphellandrene & 1526 & 3.00 \\
\hline (E)- $\gamma$-Bisabolene & 1533 & 0.64 \\
\hline (E)-Nerolidol & 1534 & 0.61 \\
\hline Elemol & 1549 & 0.20 \\
\hline Geranyl isovalerate & 1597 & 4.16 \\
\hline Cubenol & 1642 & 0.62 \\
\hline$\alpha$-Muurolol & 1645 & 0.66 \\
\hline$\alpha$-Cadinol & 1653 & 2.45 \\
\hline Ar-Turmerone & 1664 & 0.38 \\
\hline (Z,E)-Farnesol & 1697 & 12.58 \\
\hline (E,E)-Farnesol & 1772 & 0.12 \\
\hline (E,Z)-Farnesol & 1742 & 0.10 \\
\hline Tetradecanoic acid & 1771 & 0.33 \\
\hline (Z,E)-Farnesyl acetate & 1818 & 0.12 \\
\hline (E,E)-Farnesyl acetate & 1843 & 0.10 \\
\hline Hexadecanoic acid & 1973 & 1.71 \\
\hline Monoterpene hydrocarbons & & 5.79 \\
\hline Oxygenated monoterpenes & & 6.75 \\
\hline Sesquiterpene hydrocarbons & & 43.21 \\
\hline Oxygenated sesquiterpenes & & 22.34 \\
\hline Other compounds & & 12.72 \\
\hline Total & & 90.81 \\
\hline
\end{tabular}

${ }^{a}$ retention indices as determined on a DB-5 column using a homologous series of n-alkanes. 
Table 2: Percentage composition of stem and leaf oils of Dorema ammoniacum.

\begin{tabular}{|c|c|c|c|}
\hline Compound & $R I^{\mathrm{a}}$ & Stem & Leaf \\
\hline 4-methylene-5-Hexenal & 893 & 0.17 & \\
\hline$\alpha$-Pinene & 935 & - & 2.37 \\
\hline Myrcene & 991 & & 0.70 \\
\hline 6-methyl-5-Hepten-2-one & 1010 & $0 . \overline{17}$ & - \\
\hline Allyl tiglate & 1022 & 0.22 & \\
\hline p-Cymene & 1024 & 0.21 & 10.03 \\
\hline Limonene & 1031 & - & 1.06 \\
\hline (Z)- $\beta$-Ocimene & 1040 & & 7.11 \\
\hline (E)- $\beta$-Ocimene & 1050 & 0.23 & 30.94 \\
\hline$\gamma$-Terpinene & 1060 & 1.09 & \\
\hline Terpinolene & 1086 & 0.18 & 6.19 \\
\hline endo-Fenchol & 1112 & & 0.55 \\
\hline (E)-2-Nonenal & 1150 & 0.16 & \\
\hline Borneol & 1163 & & 2.59 \\
\hline Dodecane & 1199 & $0 . \overline{2} 6$ & \\
\hline trans-Carveol & 1217 & & 1.48 \\
\hline endo-Fenchyl acetate & 1220 & $0 . \overline{7} 5$ & 5.25 \\
\hline Decanol & 1272 & 0.37 & \\
\hline Bornyl acetate & 1284 & - & 0.58 \\
\hline Undecanal1304 & 0.24 & & \\
\hline 2-Undecanol & 1312 & 0.83 & - \\
\hline (E)-2-Decen-1-ol & 1375 & 1.09 & - \\
\hline 4-ethyl-1-ethylen Benzene & 1392 & 0.21 & - \\
\hline Tetradecane & 1399 & 0.31 & - \\
\hline 2-Dodecanol & 1410 & 0.39 & - \\
\hline Dodecanal1415 & 0.96 & & \\
\hline$\beta$-Caryophyllene & 1418 & $4 . \overline{3} 9$ & 0.90 \\
\hline Neryl acetone & 1434 & 0.40 & \\
\hline 2-methyl-butyl Benzoate & 1436 & & $0 . \overline{8} 9$ \\
\hline trans- $\alpha$-Bergamotene & 1438 & 0.12 & - \\
\hline 1-methoxy-Naphthalene & 1441 & 0.34 & - \\
\hline Geranyl acetone & 1453 & 3.89 & - \\
\hline Neryl propanoate & 1456 & 0.59 & \\
\hline (E)- $\beta$-Farnesene & 1458 & 0.61 & $4 . \overline{4} 2$ \\
\hline$\beta$-Acoradiene & 1464 & 0.70 & - \\
\hline$\gamma$-Gurjunene & 1473 & 0.31 & 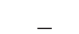 \\
\hline Germacrene D & 1480 & 0.51 & $2 . \overline{2} 9$ \\
\hline (Z)-8-Dodecen-1-ol & 1483 & 0.88 & - \\
\hline 2-Tridecanone & 1492 & 0.87 & - \\
\hline Bicyclogermacrene & 1494 & & $1 . \overline{1} 6$ \\
\hline 2-Pentadecanol & 1502 & 0.98 & \\
\hline$\beta$-Bisabolene & 1509 & 3.04 & $0 . \overline{85}$ \\
\hline$\gamma$-Cadinene & 1511 & 2.38 & - \\
\hline$\delta$-Cadinene & 1524 & 2.03 & - \\
\hline (Z)-Nerolidol & 1532 & 0.18 & $\begin{array}{l}- \\
-\end{array}$ \\
\hline$\alpha$-Calacorene & 1542 & 0.25 & - \\
\hline cis-Sesquisabinene hydrate & 1545 & 3.35 & \\
\hline Germacrene B & 1554 & & 0.64 \\
\hline Geranyl-n-butyrate & 1562 & $1 . \overline{3} 0$ & \\
\hline (E)-Nerolidol & 1564 & 5.09 & 0.57 \\
\hline Spathulenol & 1576 & 0.89 & 4.23 \\
\hline Caryophyllene oxide & 1581 & 3.41 & 0.91 \\
\hline Tetradecanal & 1598 & 3.58 & - \\
\hline iso-amyl Nerolate & 1601 & 0.25 & - \\
\hline$\gamma$-Eudesmol & 1628 & 0.17 & 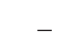 \\
\hline$\alpha$-Muurolol & 1645 & 13.68 & $2 . \overline{2} 3$ \\
\hline$\alpha$-Eudesmol & 1650 & 2.66 & - \\
\hline$\alpha$-Cadinol1653 & 4.47 & & - \\
\hline$\alpha$-Bisabolol & 1680 & $0 . \overline{4} 2$ & - \\
\hline Acorenone & 1685 & 1.59 & - \\
\hline 1,4-dimethoxy Naphthalene & 1690 & 0.75 & - \\
\hline 2-Pentadecanone & 1695 & 2.07 & - \\
\hline 2-Pentadecanol & 1705 & 0.30 & - \\
\hline$(\mathrm{E}, \mathrm{E})-\mathrm{Farnesol}$ & 1722 & 1.73 & - \\
\hline (E,E)-3,7,11-trimethyl, & 1740 & 1.02 & - \\
\hline $\begin{array}{l}\text { 2,6,10-Dodecatrienal } \\
\text { benzyl Benzoate }\end{array}$ & 1762 & 0.32 & $\ldots$ \\
\hline
\end{tabular}

\begin{tabular}{|l|c|c|c|}
\hline \multicolumn{1}{|c|}{ Compound } & $\mathrm{RI}^{\mathrm{a}}$ & Stem & Leaf \\
\hline Tetradecanoic acid & 1770 & 0.64 & - \\
Octadecane & 1800 & 0.33 & - \\
cis-9-Hexadecanal & 1812 & 0.73 & - \\
Hexadecanol & 1820 & 2.40 & - \\
6,10,14-trimethy & & & \\
l-2- Pentadecanone & 1840 & 0.17 & - \\
Pentadecanoic acid & 1868 & 0.21 & - \\
(E)-5-Octadecene & 1876 & 0.42 & $0 . \overline{7}$ \\
Hexadecanoic acid & 1976 & 6.81 & - \\
(Z,Z)-9,12-Octadecadienoic acid & 2140 & 1.22 & 69.49 \\
Monoterpene hydrocarbons & & 0.62 & 10.45 \\
Oxygenated monoterpenes & & 6.93 & 10.26 \\
Sesquiterpene hydrocarbons & & 14.34 & 7.94 \\
Oxygenated sesquiterpenes & & 37.89 & 1.86 \\
Other compounds & 89.2 & 29.42 & \\
Total & & 100 & \\
\hline
\end{tabular}

${ }^{a}$ retention indices as determined on a DB-5 column using a homologous series of n-alkanes.

Table 3 : Comparative percentage composition of the leaf, fruit and stem oils of Leutea petiolare.

\begin{tabular}{|c|c|c|c|c|}
\hline Compound & $\mathrm{RI}^{\mathrm{a}}$ & Leaf & Fruit & Stem \\
\hline$\alpha$-Pinene & 939 & 4.22 & 3.34 & 1.83 \\
\hline Camphene & 951 & 0.67 & 0.28 & - \\
\hline Sabinene & 976 & 0.66 & 0.24 & - \\
\hline$\beta$-Pinene & 980 & & 0.45 & - \\
\hline Myrcene & 991 & 2.23 & 1.07 & $1 . \overline{9} 6$ \\
\hline p-Cymene & 1024 & & 11.50 & 15.43 \\
\hline Limonene & 1028 & $9 . \overline{16}$ & 2.39 & 2.35 \\
\hline (Z)- $\beta$-Ocimene & 1040 & 7.79 & 8.32 & 4.08 \\
\hline (E)- $\beta$-Ocimene & 1050 & 10.24 & 24.63 & 10.46 \\
\hline$\gamma$-Terpinene & 1061 & 0.96 & 9.72 & 5.75 \\
\hline Terpinolene & 1086 & 13.93 & 8.28 & 10.27 \\
\hline Linalool & 1096 & & 0.11 & 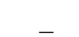 \\
\hline cis-Thujone & 1101 & $0 . \overline{25}$ & 0.13 & \\
\hline endo-Fenchol & 1111 & & & $4 . \overline{3} 2$ \\
\hline exo-Fenchol & 1114 & $1 . \overline{8} 2$ & $0 . \overline{7} 5$ & \\
\hline allo-Ocimene & 1127 & 0.88 & 0.66 & - \\
\hline trans-Sabinol & 1140 & 0.26 & 0.10 & \\
\hline 4,8-epoxy-p-Menth-1-ene & 1145 & & 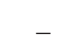 & $0 . \overline{4} 5$ \\
\hline trans-epoxy Ocimene & 1147 & $0 . \overline{1} 6$ & $\begin{array}{l}- \\
-\end{array}$ & ond \\
\hline Menthofuran & 1164 & 0.16 & - & - \\
\hline Borneol & 1165 & & - & 3.78 \\
\hline p-Mentha-1,5 dien-8-ol & 1166 & $0 . \overline{4} 0$ & $-\overline{0}$ & - \\
\hline Terpin-4-ol & 1176 & & $0 . \overline{2} 1$ & - \\
\hline p-methyl Acetophenone & 1182 & $0 . \overline{49}$ & 0.16 & - \\
\hline p-Cymene-8-ol & 1183 & 1.71 & 0.59 & $0 . \overline{9} 6$ \\
\hline methyl Salicylate & 1189 & & & 0.52 \\
\hline trans- dihydro Carvone & 1200 & - & $0 . \overline{1} 8$ & \\
\hline trans- Carveol & 1215 & - & 0.79 & $0 . \overline{4} 8$ \\
\hline endo-Fenchyl acetate & 1220 & 15.11 & 7.42 & 7.57 \\
\hline Thymol methyl ether & 1232 & & - & 0.46 \\
\hline Pulegone & 1235 & $0 . \overline{1} 9$ & - & \\
\hline Cumin aldehyde & 1239 & & & $0 . \overline{8} 8$ \\
\hline Bornyl acetate & 1285 & $0 . \overline{9} 9$ & $0 . \overline{4} 2$ & 0.62 \\
\hline Thymol & 1290 & & & 0.37 \\
\hline Carvacrol & 1296 & $0 . \overline{2} 2$ & $0 . \overline{1} 9$ & 1.19 \\
\hline Menth-1-ene-4,8-diol & 1305 & & 0.14 & - \\
\hline$\delta$-Elemene & 1336 & $0 . \overline{2} 8$ & - & - \\
\hline$\alpha$-Copaene & 1374 & 0.31 & - & - \\
\hline$\beta$-Cubebene & 1390 & 0.46 & - & \\
\hline Dodecanal & 1406 & & & $0 . \overline{3} 2$ \\
\hline$\beta$-Caryophellene & 1418 & 2.11 & $0 . \overline{8} 8$ & - \\
\hline$\beta$-Gurjunene & 1430 & 0.12 & & - \\
\hline$\gamma$-Elemene & 1432 & 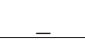 & $0 . \overline{1} 8$ & - \\
\hline
\end{tabular}




\begin{tabular}{|c|c|c|c|c|}
\hline Compound & $\mathrm{RI}^{\mathrm{a}}$ & Leaf & Fruit & Stem \\
\hline 2-methyl-buthyl Benzoate & 1436 & - & 0.46 & \\
\hline Geranyl acetone & 1453 & & & 0.8 \\
\hline$\alpha$-Humulene & 1454 & $0 . \overline{9} 1$ & $0 . \overline{1} 5$ & \\
\hline (E)- $\beta$-Farnesene & 1456 & 0.67 & 2.78 & $0 . \overline{2} 7$ \\
\hline Germacrene D & 1480 & 3.08 & 1.84 & ( \\
\hline Bicyclogermacrene & 1494 & & 0.4 & - \\
\hline trans- $\beta$-Guaiene & 1500 & $0 . \overline{2} 3$ & & - \\
\hline$\beta$-Bisabolene & 1506 & 0.77 & $0 . \overline{6} 1$ & $0 . \overline{9} 8$ \\
\hline$\gamma$-Cadinene & 1510 & 0.46 & 0.12 & - \\
\hline$\delta$-Cadinene & 1521 & 0.88 & 0.48 & - \\
\hline iso-penthyl Salicylate & 1531 & - & 0.19 & - \\
\hline Elemol & 1543 & - & 0.16 & - \\
\hline Germacrene B & 1552 & - & 0.36 & \\
\hline (E)-Nerolidol & 1564 & & 0.30 & 0.49 \\
\hline 1,5-epoxy Salvial-4(14)-en & 1565 & $0 . \overline{1} 4$ & & \\
\hline (Z)-3-hexenyl Benzoate & 1570 & 0.49 & $0 . \overline{2} 9$ & - \\
\hline Spathulenol & 1576 & 2.99 & 2.88 & $0 . \overline{6} 3$ \\
\hline Caryophyllene oxide & 1581 & & 0.60 & 0.66 \\
\hline Salvial-4(14)-en-1-one & 1589 & $0 . \overline{2} 7$ & 0.19 & \\
\hline$\beta$-Oplopenone & 1604 & 0.27 & 0.17 & - \\
\hline (Z)-Sesquilavandulol & 1606 & 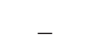 & 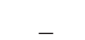 & $0 . \overline{6} 8$ \\
\hline Tetradecanal & 1611 & - & - & 0.23 \\
\hline (E)-Sesquilavandulol & 1632 & $0 . \overline{2} 9$ & $0 . \overline{2} 3$ & - \\
\hline Isospathulenol & 1642 & & 0.19 & - \\
\hline$\alpha$-Muurolol & 1644 & $2 . \overline{0} 6$ & 0.86 & $3 . \overline{4} 7$ \\
\hline$\beta$-Eudesmol & 1647 & _- & 0.18 & _- \\
\hline$\alpha$-Cadinol & 1653 & - & 0.76 & - \\
\hline Khusinol & 1674 & $0 . \overline{6} 8$ & 0.19 & \\
\hline Acorenone & 1682 & . & . & $0 . \overline{4} 0$ \\
\hline (E,E)-Farnesol & 1720 & & - & 0.38 \\
\hline benzyl Benzoate & 1730 & $0 . \overline{1} 2$ & $0 . \overline{1} 1$ & 0.45 \\
\hline $\begin{array}{l}\text { 6,10,14-trimethyl-2- } \\
\text { Pentadecenone }\end{array}$ & 1872 & 0.16 & & 0.77 \\
\hline Hexadecanoic acid & 1973 & 0.96 & $0 . \overline{2} 4$ & 9.01 \\
\hline $\begin{array}{l}\text { (Z,Z)-9,12- } \\
\text { Octadecadienoic acid }\end{array}$ & 2142 & - & - & 1.81 \\
\hline Monoterpene hydrocarbons & & 50.74 & 70.88 & 51.65 \\
\hline Oxygenated monoterpenes & & 21.11 & 11.19 & 21.88 \\
\hline Sesquiterpene hydrocarbons & & 10.28 & 6.80 & 2.05 \\
\hline Oxygenated sesquiterpenes & & 6.70 & 7.71 & 5.91 \\
\hline Other compounds & & 2.22 & 1.45 & 13.11 \\
\hline Total & & 91.05 & 98.03 & 94.06 \\
\hline
\end{tabular}

${ }^{a}$ retention indices as determined on a DB-5 column using a homologous series of n-alkanes.

\section{REFERENCES}

1. V. Mozaffarian, Flora of Iran: Umbelliferae. M. Assadi, A. A. Masoumi, M. Khatamsaz, P. Babakhanlu, Eds. Tehran: Research Institue of Forests and Rangelands, 2007.

2. K. H. Rechinger, Tetrataenium, Dorema, Leutea In Flora Iranica, Umbelliferae, No. 162, K. H. Rechinger, I. C. Hedge, (Eds.). Akademische Druck and Verlagsanstalt, Graz, Austria, 1980.

3. G. R. Amin, Popular Medicinal Plants of Iran. Tehran: Iranian Research Institute of Medicinal Plants, 1991.

4. F. A. Fluckiger, D. Hanbury, Pharmacographia: a history of the principal drugs of vegetable origin, met within Great Britain and British India. Cambridge: Cambridge university press, 1879.

5. F. Howes, Economic Botany. 4, 307 (1950)

6. A. Parsa, Medicinal plants and drugs of plant origin in Iran III. Plant Foods for Human Nutrition. 6, 137 (1959).

7. Y. Amanzadeh, Ammoniacum gum. In: Editorial Committee. Iranian Herbal Pharmacopoeia. Tehran: Ministry of Health and Medical Education Publications, 2002.

8. M. M. Karshenas, A. Arabzadeh, M. A. Tafti, G. Kordafshari, A. Zargaran, A. Mohagheghzadeh, Herbal Galen Medical Journal. 1, 78 (2013).

9. C. P. Khare, Indian Medicinal Plants: an illustrated dictionary. New Delhi, Springer, 2007.

10. A. Chevallier, The Encyclopedia of Medicinal Plants. New York, DK
Publication, 1996

11. V. P. Kumar, N. S. Chauhan, H. Padh, M. Rajani, Journal of Ethnopharmacol. $107,182(2006)$.

12. M. Rajani, N. Saxena, M. Ravishankara, N. Desai, H. Padh, Journal of Pharmaceutical Biology. 40, 534 (2002).

13. H. R. Adhami, J. Lutz, H. Kahlig, M. Zehl, L. Krenn, Scientia Pharmaceutica. 81, 793 (2013).

14. G. Appendino , G. M. Nano, D. Viterbo, G. Demunno, M. Cisero, G. Palmisano, M. Avagno, Helvetica Chimica Acta. 74, 495 (1991).

15. M. Nurmukamedova, G. Nikonov, Chemical Natural Compounds. 12, 92 (1976).

16. T.V. Bukreeva, M.G. Pimenov, Chemical Natural Compounds. 27, 638 (1991).

17. M. Iranshahi, F. Shaki, A. Mashlab, A. Porzel, L. A. Wessjohann, Journal of Natural Products. 70, 1240 (2007).

18. E. Wollen Weber, M. Doerr, A. Rustaiyan, Phytochemistry. 38, 1417 (1995).

19. M. Yousefzadeh, M. Heidari, M. Akbarpour, M. H. Mirjalili, A. Zeinali, M. Parsa, Middle-East Journal of Scientific Research. 7, 511 (2011).

20. M. Yousefzadeh, M. H. Mirjalili, N. Alnajar, A. Zeinali, M. Parsa, Journal of the Serbian Chemical Society. 76, 857 (2011).

21. S. Sajjadi, N. Ghassemi, P. Mohamadzamani, Review Arid Regions. 1, 194 (2007).

22. M. Sadeghei Takallo, S. Sajjadifar, M. Mansouji Avval, Research Journal of Pharmaceutical, Biological and Chemical Sciences. 4, 640 (2013).

23. M. R. Delnavaz, S. Tavakoli, A. Rustaie, H. Batooli, N. Yassa, Research Journal of Pharmacognosy. 1, 11 (2014).

24. H. Vanden Dool, P. D. Kratz, Journal of Chromatography. 11, 463 (1963).

25. R. P. Adams, Identification of Essential Oil Components by Gas Chromatography / Quadrupole Mass Spectroscopy. Allured Publishing Corporation, Coral Stream, Illinoise, 2001.

26. S. Masoudi, A. Esmaeili, M. A. Khalilzadeh, A. Rustaiyan, N. Moazami, M. R. Akhgar, M. Varavipoor, Flavour and Fragrance Journal. 21, 801 (2006).

27. S. Asnaashari, E. Dadizadeh, A. H. Talebpour, M. Eskandani, H. Nazemiyeh, BioImpacts. 1, 241 (2011).

28. S. Masoudi, A. Rustaiyan, N. Ameri, Journal of Essential Oil Research. 16, $143(2004)$. 